\title{
Notas sobre la noción de salud y la reflexión cultural en la psicología
}

\author{
Raúl Ernesto García Rodríguez \\ Universidad Michoacana de San Nicolás de Hidalgo \\ raulgarciar@gmail.com
}

\begin{abstract}
Resumen
Abstract

Este artículo pretende en primer lugar presentar una This article initially intends to display an abstract of síntesis de diversas posiciones teóricas acerca de la diverse theorical positions concerning the health notion idea de salud en relación a la idea de enfermedad en el in its relation to the idea of sickness within long term ámbito de la práctica en psicología a lo largo del psychological practice. Secondly, the analysis is tiempo. Posteriormente se analizan algunas categorías directed to the reflection and psychological practice of inscritas en el campo sociocultural que se vinculan a la several socio-cultural categories concerning health reflexión y a la práctica psicológica respecto a la promotion emerged from current professional practice. promoción de la salud desde el quehacer profesional Finally, the reflection is headed towards the cultureactual. Por último se hace una reflexión acerca del subjectivity plexum and its acting -throughout life style plexo cultura-subjetividad y su participación -a través and interpersonal relationships- in the configuration of del modo de vida y de las relaciones con los demás- en healthy and unhealthy situations and simoultaneously in la configuración de situaciones de salud 0 de the personal construction of the world that each being enfermedad y simultáneamente, en la construcción elaborates.

personalizada que hace el sujeto del mundo.
\end{abstract}

Palabras clave: Salud; Enfermedad; Psicología; Keywords: Health; Sickness; Psychology; Personal Cultura personal; Subjetividad; Modo de vivir.

culture, Subjectivity; Life style.

\section{Salud y enfermedad en psicología}

Diversas perspectivas epistemológicas e ideológicas han participado en la construcción de la idea de salud y enfermedad mentales. Pensadores de la era moderna y en particular del siglo XVIII concibieron por ejemplo la noción misma de enfermedad mental y asumían en ella la presencia de alteraciones bioquímicas subyacentes en el individuo, con lo cual se inauguraba el llamado modelo médico de la enfermedad mental. Durante el siglo XIX y principios del siglo XX surge y se desarrolla la concepción de que las alteraciones de la conducta serían el resultado de una confrontación dinámica de naturaleza psíquica en el individuo para satisfacer deseos instintivos por un lado, y por otro, adaptarse a las exigencias y demandas de carácter socio cultural. Esta vertiente de pensamiento, que se sistematizó más tarde en el psicoanálisis, inauguraba de hecho el llamado modelo psicológico de la enfermedad mental. Es en realidad hasta los años sesenta del siglo XX que desde la psicología, se aborda sistemáticamente para su estudio el conjunto de determinantes 
ecológicas (ambientales y sociales) del trastorno mental y se piensa la enfermedad más en términos de prevención poblacional, que de curación individual, proyectándose así el llamado modelo psicosocial de la enfermedad mental. Con él se enfatiza el carácter activo de la comunidad y de los individuos en la promoción de la salud psicológica, reconocida en un funcionamiento autónomo y pleno de tales personas y grupos. Se asume una actitud horizontal, igualitaria y dialógica en el trabajo profesional en contraposición a la actitud vertical, jerárquica y prescriptiva del modelo médico ortodoxo, y en definitiva, más que promover la reparación terapéutica retrospectiva e individualizada de los efectos del trastorno psicológico ("waiting mode") se busca la prevención y la confrontación activa de las fuentes de tal trastorno ("seeking mode"); (A. Sánchez, 1991).

De manera similar, la noción de salud en la psicología ha implicado diversos modelos o concepciones básicas de comprensión e interpretación: una de las más elementales es aquella que concibe la salud mental como ausencia de trastorno o enfermedad mental. Va de la patología severa a un "punto cero" que es la ausencia de síntomas psicopatológicos, lo cual por supuesto no significa un "rendimiento óptimo" del sujeto. En esta concepción se busca eliminar del individuo el trastorno y sus síntomas y restaurar un nivel "razonable" de funcionamiento personal. Se ha concebido también la salud psicológica como normalidad estadística de los atributos psíquicos. Es decir, la enfermedad mental se asume por la desviación individual del sujeto respecto a los valores promedio. De igual forma se ha identificado la noción de salud psicológica con cierta normalidad social que implica la aceptación por parte del individuo de normas y valores sociales, es decir, un ajuste del sujeto a la sociedad para la cual -según esta idea- cualquier disconformidad o desviación respecto a tales normas y valores puede ser signo de patología. En efecto, la noción de "normalidad" ha sido vinculada tradicionalmente a la presencia de salud psíquica, tanto desde el punto de vista de la ubicación del sujeto en manifestaciones psicológicas promedio, como por la presentación de conductas socialmente aceptadas. Es pertinente señalar que en el debate teórico al respecto, se ha planteado que en ningún caso, el concepto de lo "normal" podría identificarse de manera plena como sinónimo de salud psicológica por quienes aducen que los criterios estratégicos de integración saludable se ubican más bien, en la dimensión individual subjetiva de la persona en cuestión. Como afirma Fernando González Rey: "lo sano alcanza una definición individual que, aún cuando refleje regularidades generales, éstas no se pueden definir como criterios estandarizados para ubicar a cada uno de los individuos sanos" (F. González, 1993 p.82).

Otro criterio es el que asume la salud psicológica como proceso, es decir, no en términos de conducta, estado o estructura psicológica estática, sino como proceso dinámico que se desarrolla y se relaciona interactivamente con su entorno. Este criterio incluye una perspectiva evolutiva (la salud como resultado final de un proceso vital donde participa lo biológico, que se deriva en etapas) y la perspectiva ecológica (la salud como resultado de la interacción del individuo con su contexto de sistemas socioculturales). Desde esta concepción, la salud no se define en el individuo per se, sino en cualquier caso por referencia obligada al entorno total. La salud -afirma González Rey al respecto- "es un complejo proceso cualitativo que define el funcionamiento completo del organismo, integrándose de forma sistemática lo somático y lo psíquico, formando una unidad donde la afectación de uno actúa necesariamente sobre el otro. Pienso que el concepto de salud debe considerarse más como proceso que como producto". (González, 1993 p.8).

La salud psicológica por último puede verse también como utopía o estado ideal, esto significa que la salud se mueve desde un "punto cero" (ausencia de trastorno y síntomas) hasta un polo ideal aunque perseguible de funcionamiento e integración óptima en la persona. 
En efecto, tanto la noción de salud psicológica como la de enfermedad o trastorno mental presentan un extenso carácter polisémico y han sido objeto de representación polimórfica y de interpretación diferenciada en diversos contextos histórico-sociales y filosófico-científicos. En todo caso, cualquier aproximación al concepto de salud y enfermedad en psicología, bien podría considerar aspectos tales como el estado de bienestar general; los recursos sociales y personales-subjetivos (por ejemplo información y destrezas vitales) para la interacción del individuo con el entorno tanto en el presente como en el futuro; las configuraciones personológicas; los sistemas de actitudes habituales del individuo (tanto de carácter adaptativo como confrontativo); las capacidades potenciales y desarrolladas y -de manera significativa- la cultura contextual propia del individuo y la forma en que tal individuo la personaliza, la produce y la proyecta. Así, la noción de salud en la psicología estaría relacionada con varios aspectos: base hereditaria, adecuado desarrollo biológico y neuronal, educación, relaciones familiares, condiciones y posibilidades de vida social comunitaria, nivel de realización personal en el medio socio-laboral, relación entre las capacidades del sujeto y las exigencias económicas culturales e interpersonales, entre otras. Es por eso que para la comprensión de estos procesos puede ser necesaria la convergencia de diversas perspectivas teóricoinvestigativas que van desde el análisis psicológico de las dimensiones de la afectividad y sus correlatos neurofisiológicos, hasta las funciones interpersonales y subjetivo-culturales que se constituyen como elementos socialmente asociados al malestar o al bienestar interno.

Probablemente una de las elaboraciones teóricas más importantes al respecto se encuentra vinculada a la idea de la llamada Salud mental positiva que se presenta como un concepto afirmativo y potenciador cuyas líneas de análisis tienen afinidad con la obra de pensadores como Marie Jahoda, Gordon Allport y Strupp Hadley, junto a otros psicólogos de la llamada "tercera fuerza" (Sánchez, 1991). Estos autores desde la psicología social y desde la psicología de la personalidad, incluyen en sus elaboraciones diversos criterios que implican el desarrollo individual y comunitario de las personas, el ámbito subjetivo en términos de personalidad, actitudes y emocionalidad y diversas capacidades de diálogo e integración constructiva respecto al ambiente. En el caso particular de Jahoda, se valora con carácter explícito el hecho de que las normas del comportamiento saludable varían con el tiempo y la cultura, siendo imposible por tanto fijar, en este sentido, preceptos absolutamente "objetivos" o "independientes" precisamente de la percepción social y de la cultura, aunque la salud psicológica se viva como un atributo personal e individualizado. Se admite además que no puede existir un concepto comprensivo totalmente aceptable de salud psíquica y más bien deben considerarse elaboraciones multicomponenciales evitando cualquier visión unidimensional $u$ homogénea.

La salud psicológica implicaría en una dinámica flexible, no sólo la sensación de bienestar interno y la presencia de motivaciones e intereses más o menos estables en el individuo, sino además la capacidad de autorregularse de manera transformadora y productiva y de proyectarse en esta dirección hacia el futuro, todo lo cual en definitiva se relaciona con la presencia de un modo de vida culturalmente construido. No obstante, "la salud es una expresión plurideterminada y su curso no se decide por la participación activa del hombre de forma unilateral. Este es uno de los elementos que intervienen en el desarrollo del proceso, pues muchos de los factores de salud son ajenos al esfuerzo volitivo del hombre". (González, 1993 p.8).

Por otro lado, la noción de "enfermedad psicosomática" pierde vigencia en la investigación en tanto se asume cada vez con más frecuencia que todas las enfermedades implican participación psicosomática. Se afirma que lo psíquico siempre interviene de diversas formas en la presencia y el 
curso de la enfermedad. En esta lógica, el ámbito psicológico repercute en el ámbito somático, no de manera directa en la aparición del síntoma, sino a través de la generación de expresiones psicológicas disfuncionales que alteran la dimensión bioquímica y fisiológica del organismo incluyendo el aspecto inmunológico. En esta dirección, R. Lazarus y S. Folkman plantean: "Ampliar el concepto de psicosomático desde un grupo específico de dolencias tales como úlceras e hipertensión, hasta el concepto general de que toda enfermedad podría tener una etiología psicosocial dentro de un sistema multicausal, ha estimulado la consideración de la respuesta inmune como posible factor incluso en la aparición de procesos neoplásicos, trastornos sumamente alejados del significado original del psicosomático." (R. Lazarus, S. Folkman, 1991 p.33). Así también se señala que por ejemplo los llamados mecanismos neuróticos de la personalidad -como formas inadecuadas de regulación psicológica- afectan la salud somática. Dichos mecanismos estarían relacionados con características tales como la rigidez, la ausencia de perspectivas y elaboraciones futuras en el sujeto -anquilosamiento de la dimensión del pasado en el individuo-, dificultades en la reconceptualización o revaloración de situaciones emocionales y experiencias e incapacidad de autodeterminación y ajuste independiente ante las contingencias vitales, todo lo cual habría de incidir integralmente en el rendimiento fisiológico de la persona.

A su vez, el ámbito somático forma parte también de la salud psíquica: Dado el carácter sistémico de las conductas de salud, cualquier disfunción del organismo biológico tendrá resonancia psíquica, no necesariamente inmediata, independientemente de la conciencia que el sujeto tome de ello. En este sentido, señala González Rey: "Así el proceso de obesidad, que sin duda daña el organismo, puede reflejarse a nivel psicológico en un bajo nivel de autocontrol del sujeto en la regulación alimenticia, lo cual, si bien no es vivenciado negativamente por el sujeto, sí daña su salud física y a la vez mental, en tanto que la inadecuada regulación de esta esfera puede tener repercusiones en el tono, la energía y la capacidad del sujeto en otras esferas vitales para él." (González, 1993 p.14). De forma específica la enfermedad somática afecta lo psicológico tanto de manera directa por ejemplo cuando se altera la propia base biológica de lo psíquico -enfermedades del sistema nervioso y endocrinocomo de manera indirecta a través de las configuraciones subjetivas e imágenes autovalorativas que el individuo construye respecto a su enfermedad. De nuevo, González Rey comenta: "En muchas ocasiones, sobre todo en enfermedades de mal pronóstico, cuya aparición se asocia con la muerte como el cáncer y el SIDA, los individuos resultan aplastados por la expectativa de pérdida de vida, desorganizándose sus distintos sistemas de sentidos psicológicos en la personalidad. La pérdida de intereses y objetivos esenciales en las esferas que resultaban más relevantes para él, tiene efectos psicológicos verdaderamente dañinos para el proceso de enfermedad." (González, 1993 p.15).

Respecto a la mediatización psicológica en el proceso de enfermedad somática debe mencionarse por la difusión alcanzada, el enfoque de Friedman y Rosenman quienes en 1974 intentaron definir patrones comportamentales estables -a partir de una serie de características y rasgos psicológicosrelacionados estadísticamente con trastornos cardiovasculares importantes (M. Friedman, R. Rosenman, 1974). Los autores tipificaron los llamados patrones A y B sobre la base de investigaciones realizadas en tal sentido. Esta reflexión supone que las personas pertenecientes al tipo o patrón A serían más vulnerables a desarrollar determinadas enfermedades cardiovasculares en tanto son aquellas que viven con una noción extrema de la urgencia del tiempo, tienen multiplicidad de metas, proyectan una implicación exagerada con sus actividades, presentan agresividad y competitividad permanentes y son por tanto irritables, impacientes y tensos. Las personas del tipo o patrón B tendrían menor riesgo cardiovascular precisamente por no reunir los rasgos mencionados. El problema fundamental de estos trabajos es su carácter descriptivo (delineamiento de 
comportamientos típicos) y cuantitativo (intento de estandarización de las categorías a partir de las diferencias en las actividades descritas) y el hecho de que no desarrolla una proyección explicativa que profundice la teorización sobre la personalidad como concepto psicológico en su relación con tales efectos somáticos. Además, como sostiene Francisco Morales Calatayud: "(...) los resultados de las investigaciones han sido contradictorios, pues han evidenciado que muchas personas con el patrón A no desarrollan la enfermedad cardiovascular, mientras que otras con el patrón B la desarrollan, así como que hay muchas personas que pueden considerarse tipos mixtos (...) Por eso, tampoco este patrón (el A) puede ser asumido de manera simplista como factor psicológico de riesgo, pero nos es útil como orientación general para el estudio de los ambientes sociales y los tipos de comportamientos frecuentes que en éstos se observan, y como una guía más (no rígida) para la interpretación de la interacción del individuo con el ambiente." (F. Morales, 1999 pp.97-98)

Metodológicamente, la evaluación de presencia del patrón A se ha realizado por lo general con el Cuestionario de Actividad de Jenkins (C. Jenkins, R. Rosenman, J. Zyzanski, 1974) de carácter estructurado, con lo cual se presentan múltiples limitaciones: su connotación experimental de laboratorio, su visión descriptiva y cuantitativa que no involucra procesos personológicos implicados en las situaciones presentadas y la no consideración de las diferencias individuales dentro de los propios sujetos exponentes de uno u otro patrón. Por todo esto, existen cuestionamientos sobre la connotación del patrón A como elemento de riesgo para los trastornos cardiovasculares, sobre todo en el sentido de que no sería el patrón A por sí mismo el causante de las afectaciones somáticas, sino el patrón A combinado con los sentidos personológicos y los procesos de evaluación y afrontamiento específicos que cada sujeto afectado le ha otorgado a sus interacciones con el mundo.

La salud psicológica entonces podrá considerarse en una dinámica permanente de inclusión que implique el aspecto somático, la personalidad individual y su participación irrepetible en la configuración psicopatológica, los elementos interpersonales y sociopsicológicos en su significación específica y la cultura misma, que se elabora de manera única en cada sujeto. Se abre la posibilidad de concebir los procesos de salud y enfermedad mental atendiendo a la complejidad que representa el papel activo de la regulación interna en los mismos, así como la historia personal de cada individuo que promueve sentidos psicológicos diferenciados, es decir, considerar toda conducta en su contexto y en relación a los aspectos personológicos que están expresándose en ella.

\section{Aspectos sociales e interactivos}

El proceso de salud en el ámbito psicológico implica una trayectoria social, histórica y cultural que se personaliza en el individuo y cuyas manifestaciones cualitativas se modifican permanentemente con el desarrollo del hombre. Es necesario contribuir a la construcción interpretativa de los aspectos participantes desde la asunción de los dinamismos psicológicos sociales y culturales en permanente relación. En este sentido diversas investigaciones han establecido un vínculo entre la salud psicológica y diferentes indicadores de la posición social, por ejemplo, la clase social, el género y la situación laboral entre otras. De esta manera la salud no se analiza desde una perspectiva médica sino desde una perspectiva que la interpreta como el proceso de ofrecer respuesta adaptativa y transformadora a las demandas y exigencias del entorno (concepción sociopsicológica de la salud mental). En efecto, la salud mental puede verse como la capacidad de participar en la configuración de significados y sentidos sociales y por supuesto en la transformación y recomposición de la realidad social. La labor del psicólogo puede y tal vez debe encaminarse hacia tales intentos. Favorecer la 
modificación de asunciones culturales que obstaculizan el despliegue de conductas productivas y de creatividad. Por tanto, la concepción del diagnóstico y del tratamiento de tales problemas ha de tener una orientación relacional y procesual. Las estrategias de "afrontamiento" de la enfermedad mental han de suponer esfuerzos no sólo cognitivos y comportamentales sino también interactivos y de recomposiciones subjetivo-culturales que permitan ya sea reducir, tolerar, enfrentarse y/o transformar demandas externas e internas actuantes en el curso de las transiciones de desintegración para el individuo. En esta lógica, la persona ha de tener la posibilidad de adquirir recursos psicológicoculturales no sólo en función de promover un momento adaptativo en su relación con el mundo, sino además, de potenciar una proyección transformadora y reconstructiva del propio entorno que se personaliza en el sujeto.

Por otra parte diversas investigaciones han señalado que los trabajadores manuales no cualificados presentan una importante incidencia de alteraciones psicológicas, con lo cual se subraya la idea de que el "descenso" en el bienestar psicológico de los obreros puede ser consecuencia compleja de una situación económica desfavorable y de insuficiencias cualitativas en las integraciones y proyecciones culturales de tales individuos. Así, el individuo que utiliza su tiempo de vida en sobrevivir tendrá mayor exposición a acontecimientos tensionantes y presentará además determinada vulnerabilidad ante los mismos, lo cual puede implicar de diversas maneras, la aparición de trastornos o desintegraciones psicológicas. En ésta lógica, se ha planteado, por ejemplo, la importancia del estatus de empleo y las condiciones de trabajo en la configuración del desarrollo saludable de la subjetividad en el individuo. Se presentan efectos psicológicos negativos en empleos precarios o inestables producto frecuentemente del alto grado de incertidumbre respecto al futuro de la persona. De manera similar, intervienen las condiciones físicas, organizativas e interactivas en que se desempeñan los puestos de trabajo; la oportunidad de control; la adecuación entre exigencias del puesto y las capacidades de individuo que lo realiza; las relaciones interpersonales; el salario; la seguridad física entre otros.

Así, la posición social se asocia a la experiencia emocional subjetiva de los individuos. En este sentido afirman Mirowsky y Ross: "Sin la habilidad o el deseo de enfrentarse con las abrumadoras fuentes de estrés presentes en los niveles más bajos de la jerarquía social, los problemas no resueltos derivados de una situación de pobreza y bajo nivel educativo se acumulan. Ésta combinación de mayores problemas y menores recursos para enfrentarse a ellos incrementa el deterioro psicológico de las personas más desfavorecidas." (J. Mirowsky, C. Ross, 1989 p.95). Ahora bien, es pertinente señalar que -según la reflexión de Cochrane y Sobol- (R. Cochrane, R.Sobol, 1980) el deterioro psicológico puede ser concebido como un mecanismo de respuesta de carácter adaptativo al entorno cuando no aparecen otro tipo de alternativas disponibles, lo cual no constituye un estado necesariamente patológico.

Por su parte, Alvaro, Torregrosa y Garrido afirman: "La desigual distribución del bienestar emocional es representativa de las diferencias en calidad de vida asociadas con diferentes posiciones sociales, mostrándonos la conexión entre realidades externas de desigualdad objetiva y la experiencia subjetiva de deterioro emocional. El mapa social de desigualdades en posición social es, en términos probabilísticos, isomórfico con un mapa subjetivo de equilibrio psicológico." (J. Alvaro, J. Torregrosa, A. Garrido, 1992 p.26). La salud mental puede pensarse entonces como un indicador de desigualdad psicosocial. Así, resulta necesario trabajar en un modelo que implique -más que los preceptos del modelo médico de tratamiento sintomático- las características sociales del entorno y las relaciones 
interpersonales vistas como producto cultural activo. Se hace necesario promover en efecto una "psicología social de la salud mental" en ámbitos comunitarios.

Aunado a esto, Agustín Ozámiz (A. Ozámiz, 1992 pp.73-74) destaca el desempleo, la internacionalización de la información, la urbanización extrema con sus secuelas de soledad, es decir, la pérdida o disminución de vínculos grupales, la tecnificación del entorno con la consecuente alteración de las coordenadas del tiempo y del espacio, el cambio en la importancia del individuo en la sociedad, de nuevo la soledad a pesar de muchas aparentes relaciones, la sensación de impotencia ante el cúmulo de información de los medios de comunicación de masas, todo ello, como situaciones que en la actualidad pueden estar vinculadas a la ansiedad y al temor de muchas personas.

En esta dirección, la noción de sucesos vitales o acontecimientos vitales se utiliza para operacionalizar la investigación sobre cómo las situaciones sociales influyen en la aparición y desarrollo de desintegraciones psicológicas. Lo fundamental aquí es resaltar que cualquier suceso o acontecimiento vital es importante para el sujeto sólo en la medida en que dicho sujeto lo incorpora de forma significativa al escenario de sus vivencias personalizadas. Es decir, el acontecimiento nunca tiene valor per se sino sólo a través de la experiencia emocional del individuo que lo vive de manera única y le asigna unos u otros valores de relación. En este sentido afirma Alfred Schutz: "Las diferencias temporales y biográficas en los flujos subjetivos de experiencia, codeterminan una perspectiva individual en estructuras de significatividades que están, por lo demás, estrictamente 'socializadas'; estructuras de significatividades sobre cuya base las experiencias son sedimentos en el acervo subjetivo de conocimientos (...)" (A. Schütz, 1973 p.293). En efecto, los sucesos vitales que se personalizan, influyen en el pensamiento, en los ordenamientos cognitivos del sujeto, en sus relaciones y conductas y eventualmente en sus descomposiciones subjetivas. Resulta interesante destacar que se han realizado estudios de diversas patogenias -en particular en las tradicionalmente llamadas enfermedades psicosomáticas- relacionados con situaciones o acontecimientos vitales (G. Brown, T. Harris, 1989). Se subraya que tales sucesos influyen en el proceso de salud-enfermedad del individuo, a través de los significados (es decir de la percepción de significación) asociados a ellos. Se trata de la posibilidad de comprender los sentimientos individuales ubicándolos en el contexto de significación en que tienen lugar, y además, de analizar el proceso por medio del cual el sujeto interactúa en su entorno y realiza una determinada apreciación cognitivo-emocional que tiene incidencia clave en el ámbito neuroendocrinológico y fisiológico de su propia existencia. Así, la personalidad se ubica en el centro de la evaluación situacional que hace el individuo del acontecimiento, y que se traduce en afectaciones de un sentido o de otro. En otros términos, la personalidad se organiza situacionalmente e involucra constructos que el individuo elabora en función de sus proyecciones y condiciones interactivas, y desde luego, cada individuo puede contar o no, con recursos psicosociales -estrategias de afrontamiento, apoyo social- que influyen en la vulnerabilidad ante uno u otro acontecimiento. En cualquier caso -en el ámbito de la atención psicológica- resulta de importancia capital, la comprensión individualizada de la situación del sujeto, siempre con carácter contextualizado respecto a su cultura social e individual.

Respecto a los eventos vitales considerados negativos se puede insistir en que ningún acontecimiento por relevante que parezca tiene capacidad desestructurante por si mismo (para el individuo que lo experimenta) al margen del sentido psicológico específico que adquiera en el sujeto. Tradicionalmente se han realizado investigaciones sobre los eventos vitales utilizando cuestionarios que otorgan de manera estandarizada valores numéricos absolutos -indicadores de su "potencial 
estresante"- a unos u otros sucesos de la vida, con lo cual cuantitativamente se valora -según tales instrumentos- la posibilidad del individuo de acceder a un episodio de distrés. Se desconoce con tales procedimientos, la especificidad irrepetible y la individualización subjetiva que de cada suceso realiza la persona en su contexto interactivo. Es decir, no se puede establecer un vínculo directo entre la ocurrencia de determinado suceso vital y el surgimiento de distrés, pues como se ha expresado, en el proceso van a intervenir diversos momentos mediatizadores.

En la óptica de enfatizar el ámbito interactivo en la comprensión y análisis de la salud psicológica, puede destacarse el trabajo de David Good (D. Good, 1992) que se detiene en el estudio de los trastornos del pensamiento y del lenguaje de los esquizofrénicos pero con la perspectiva de atender significativamente el papel que el contexto conversacional juega en la inteligibilidad del discurso esquizofrénico. Así, Good plantea que a pesar de la dificultad para distinguir trastornos en forma y contenido de este tipo de lenguaje, la variabilidad en la aparición de tales trastornos del lenguaje debe relacionarse con secuencias conversacionales particulares, y que mientras ciertas capacidades de la conversación necesarias para la cooperación interactiva son mantenidas por el esquizofrénico, otras no se corresponden con el curso de la comunicación. Lo importante en este caso es que el autor mencionado ofrece la posibilidad de desplegar un estudio del lenguaje esquizofrénico a través de la dimensión interactiva, que analiza el discurso lingüístico en relación al contexto comunicativo y no de manera aislada tal y como se han realizado estudios anteriores. En efecto, en las investigaciones acerca del lenguaje esquizofrénico se ha hecho énfasis en el trastorno general de la capacidad cognitiva, o en el posible déficit selectivo del esquizofrénico respecto a la capacidad de organizar el material lingüístico en el nivel discursivo y pragmático, no obstante, la idea de que la génesis y el despliegue de la esquizofrenia se relaciona con problemas interactivos, frecuentemente ha sido subestimada, a pesar de ser precisamente en la interacción donde ocurren buena parte de los problemas de la esquizofrenia. Good afirma que en el área del lenguaje aparentemente desordenado, es mucho mayor la significación de los detalles comportamentales y del contexto conversacional. El "desorden" puede ser más "aparente" que "real" producto de la falsa apreciación del analista o de la incapacidad de los participantes para conseguir la contextualización apropiada. Señala Good: "Siempre que decimos algo, diseñamos una locución para un interlocutor concreto y un contexto determinado. A pesar de que las emisiones verbales se diseñen en función del receptor, los demás oyentes a menudo pueden inferir un contexto plausible y, de ese modo, interpretar lo que está diciendo. No obstante, también es posible que el contexto inferido no sea el correcto (...)" (Good, 1992 p.174). El problema es que cuando se asume que una persona está "loca" y que, consecuentemente su discurso más que querer comunicarnos algo simplemente "informa" de sus disfunciones psiquiátricas, las posibles interpretaciones de lo que dice no llegan a considerarse realmente. Pero en este caso -como llegan a afirmar Rochester y Martin- (Rochester, Martin, 1977) la situación fundamental no es la presencia de un paciente "desconcertado" o de un "trastorno del lenguaje", sino de un psiquiatra desconcertado. En definitiva, queda clara la necesidad de analizar detalladamente y entender todo el contexto conversacional, es decir, de contextualizar la locución en función de ampliar las posibilidades constructivas de inteligibilidad e interpretación del lenguaje aparentemente desordenado en el ámbito de la atención psicológica y concebir al sujeto como alguien que desarrolla producciones subjetivas propias y distintas a las de uno mismo, y que implica necesidades interpretativas específicas, todo esto en una actitud dialógica.

Por otro lado, el estrés, es un término empleado sistemáticamente desde 1936 por Hans Selye quien lo definió como un conjunto interrelacionado de reacciones en el individuo ante cualquier estímulo nocivo, incluidos los psicológicos (Lazarus, Folkman, 1991). Selye relaciona el estrés 
fundamentalmente con respuestas fisiológicas a demandas del entorno, y lo ubica como "Síndrome general de adaptación". Posteriormente, otros autores -y de manera muy significativa el propio R. Lazarus- dimensionan el fenómeno del estrés en una perspectiva psicológica. Lazarus enfatiza la noción de evaluación psicológica con lo cual asigna un papel mediatizador al sujeto en el proceso tensionante y ve en el estrés una relación particular entre el individuo y el entorno, la cual es evaluada por la persona como amenazadora o que puede desbordar sus propios recursos de afrontamiento, y con esto, poner en riesgo su bienestar y su tranquilidad. Es posible pensar el estrés como una configuración subjetiva de cierto nivel de generalidad en la persona que implica una elaboración más o menos activa del individuo, es decir, la construcción específica de reflexiones y vivencias de tensión a partir de la personalización de la cultura en que ese sujeto vive, y de las actitudes y comportamientos de ese individuo hacia su mundo sociocultural. Fernando González Rey señala: "Creemos que un aspecto esencial, disparador de la reacción integral que presupone el estrés, es la calidad de las emociones que el sujeto experimenta, las cuales están esencialmente determinadas por el proceso personológico que activamente mediatiza el mundo externo e interno del sujeto y que descansa en la configuración psicológica de la personalidad." (González, 1993 p.71). Es pertinente señalar en acuerdo con este autor, que el estrés psicológico puede sostenerse en un espacio de adecuación positiva cuyo advenimiento se relaciona con una integración psicosomática expresada por el individuo en acciones y relaciones que potencien intereses y motivaciones significativas para el propio sujeto. De esta forma la conducta tensional se organiza personológicamente y se expresa hacia la productividad o la transformación positiva.

Ahora bien, el estrés como tensión psicológica integral y más o menos estable, puede convertirse en distrés cuando adquiere connotaciones profundamente perjudiciales para el sujeto. De esta forma, la repercusión fisiológica del distrés se relaciona con trastornos de riesgo para diferentes enfermedades, entre ellas la hipertensión arterial. En el distrés o estrés negativo también se reconoce una etiología múltiple y sistémica, y también se le relaciona con los recursos personológicos del sujeto para asumir y resolver situaciones significativas que lo agobien. Además, el distrés puede ser resultante no sólo del exceso o sobrecarga de actividades en relación con el sujeto que las vive, sino también de la ausencia de estimulación significativa y de actividades que involucren personológicamente al individuo, es decir, el distrés puede aparecer de la carencia de sentidos subjetivos activadores hacia la realización de unas u otras tareas, y de la imposibilidad del sujeto de formular planes y dispositivos de acción gratificantes para su futuro inmediato y mediato. En esta circunstancia, aunque el ritmo y organización de las actividades sea holgado para la persona, ésta podrá vivir importantes tensiones internas que provoquen la aparición del distrés.

Así, el carácter positivo o negativo del estrés como tensión personal, estará relacionado tanto con la implicación del sujeto como con las posibilidades de expresión que éste tiene, respecto al conjunto de actividades y relaciones en las que está inmerso, y no sólo con la calidad y la cantidad de las actividades en sí mismas. Otros aspectos que pueden intervenir a favor o en contra de la aparición del distrés, son el apoyo social que reciba el individuo, el grado de control que percibe el sujeto sobre sus actividades (vivencia de la relación entre esfuerzo y resultado) y los propios eventos vitales significativos.

Según Lazarus (Lazarus, Folkman, 1991) el estrés sería la resultante de una apreciación cognitiva que realiza el individuo del desequilibrio entre sus recursos y capacidades y las demandas de los acontecimientos o situaciones que lo agobian. Esta concepción es relacional y es orientada al proceso, lo cual significa que la desintegración psicológica se concibe no tanto como una propiedad 
de la persona o del entorno en sí mismos (no es ni "estímulo" ni "respuesta" de forma aislada) sino que constituye una relación particular entre la persona y su circunstancia, relación que es dinámica, cambiante e interactiva.

Por otro parte, resulta interesante observar que según las investigaciones de Páez, Adrián y Basabe (D. Páez, J. Adrián, N. Basabe, 1992) los estados de ánimo negativo se asocian a un estilo de pensamiento convergente, reduccionista y conservador (recuérdese que buscar explicaciones se relaciona generalmente con hechos negativos o de fracaso, incluso -al menos en lengua inglesaexiste mayor número de verbos que describen e interpretan comportamientos sociales de connotación emocional negativa) pero también de contraste y análisis de la realidad, así como de readecuación de expectativas. En sentido inverso, los estados de ánimo positivos se asocian a un estilo de pensamiento divergente, creativo e innovador y se relaciona con nuevas posibles formas de adaptación y transformación respecto al entorno. La cuestión es que la visión optimista del mundo y de uno mismo, se construye y se mueve culturalmente, de ahí que la salud psicológica y el desarrollo de las potencialidades individuales pueda plantearse dinámicamente desde una óptica cultural y en particular, desde el análisis de cómo los individuos personalizan y proyectan la cultura en que viven.

Por otro lado, el llamado apoyo social es un "constructo multidimensional" que implica distintos aspectos, fundamentalmente, provisión de apoyo emocional, apoyo material y apoyo informacional. Ana Barrón citando a Lin y colaboradores (A. Barrón, 1992 p.224) comenta que el concepto de apoyo social es definido como las provisiones instrumentales y/o expresivas, reales o percibidas, aportadas por la comunidad, redes sociales y amigos íntimos. Por su parte, Michael Argyle (M. Argyle, 1992) pondera básicamente las relaciones de amistad, las relaciones maritales y familiares y las relaciones laborales como espacios clave en la posible configuración del apoyo social para el sujeto.

Se piensa que el apoyo social opera a través de efectos directos e indirectos en el individuo. Esto significa que el apoyo social, en primer lugar, fomentaría la salud, el bienestar y la integración psicológica por sí mismo, es decir, independientemente de los episodios estresantes, ya que proporciona al sujeto un conjunto de identidades sociales que ofrecen significados vitales, previenen ansiedad y guían conductas; se presenta como fuente de autoevaluación positiva para el individuo (autoestima) y constituye una base para crear una sensación de control satisfactorio del entorno por parte del sujeto. Afirma Barrón: "La percepción de ayuda potencial provoca un aumento general del estado de ánimo positivo, de la autoestima, de la estabilidad, de la propia situación vital, sensación de control sobre el ambiente, reconocimiento de la valoración personal etc." (Barrón, 1992 p.225). En segundo lugar, el apoyo social tendría un efecto protector respecto a los efectos desestabilizadores y patogénicos de los "estresores sociales", y por tanto, significaría de hecho, una implicación terapéutica potencial. Desde esta perspectiva, han surgido precisamente los llamados grupos terapéuticos de apoyo o de "autoayuda". Han surgido además distintas estrategias interventivas que fomentan el apoyo social a nivel individual, grupal y comunitario (M. Roca, M. Pérez, 1999). Sigue por tanto vigente la reflexión de que el ambiente social e interpersonal y sus constructos culturales e interactivos, tienen una connotación etiológica tanto en el desarrollo de las potencialidades subjetivas positivas, como en sus derivaciones de desintegración y de hipertrofia.

El apoyo social puede presentarse de distintas maneras: Weiss menciona vinculación, integración social, posibilidad de nutrición, reafirmación del valor de uno mismo, sensación de alianza segura y posibilidad de obtener consejo (R. Weiss, 1974). Por su parte Schaefer y colaboradores (citados por Lazarus y Folkman, 1991) hablan de apoyo emocional, apoyo tangible y apoyo informacional. En cualquier caso, el apoyo social podría considerarse un recurso para el individuo, más o menos 
disponible del entorno, que también se cultiva y utiliza de una u otra forma. El apoyo social puede amortiguar o atenuar efectos de la tensión en el individuo, y además, puede convertirse en un espacio y una forma de interacción que resulte beneficiosa en función de favorecer la integración psicológica productiva en la persona, lo cual deviene generalmente en la proyección de una vivencia de felicidad.

Otro aspecto relacionado con la salud psicológica y que se aborda con cierta frecuencia en la investigación es el análisis del llamado estilo de vida. Con este término se hace referencia al conjunto de actitudes y comportamientos específicos que un individuo proyecta y realiza consistentemente en su vida cotidiana y que tiene una relación más o menos directa con la potenciación de su salud o el riesgo de contraer enfermedad. En esta categoría se incluyen conductas tales como el fumar, el consumir alcohol y/o drogas, el control alimenticio, la realización de ejercicios físicos, el manejo del estrés entre otros. Así, el estilo de vida como conjunto de hábitos personales puede ser modificado positivamente por el sujeto a través del aprendizaje. No obstante, en esta dimensión tampoco se puede eludir la implicación y las connotaciones subjetivas particulares que el individuo produzca al momento de asumir una $u$ otra conducta de salud o enfermedad. Además, la significación más o menos saludable de una conducta específica es relativa al ámbito social y cultural en el que vive el sujeto. Habría que plantearse -más que la promoción individual de hábitos nuevos- la revisión, y de hecho, la eventual y paulatina recomposición de la cultura contextual, valores, ideas, costumbres y normas de consumo que interioriza y exterioriza el individuo inmerso en su comunidad. Como afirma Francisco Morales Calatayud: "La interpretación con una orientación puramente biomédica del estilo de vida, eclipsa el análisis de los factores de contexto." (Morales, 1999 p.89).

También el conjunto de creencias de salud o enfermedad de las personas, su estructuración y proyección, pueden influir en las actitudes que potencian la integración o la desintegración psicológica. En este proceso se involucra el valor que cada individuo produce respecto a la probabilidad de que determinadas acciones o condiciones le permitan mejorar su salud en general y su bienestar interno. Así el individuo es más o menos susceptible en términos de enfermedad a determinadas condiciones del entorno, en dependencia de las percepciones subjetivas -creenciasque dicho individuo tenga respecto a tales condiciones, su "severidad", su "importancia", sus posibles "beneficios" o "daños" etc. En base a sus creencias el sujeto también tomará unas u otras decisiones en relación a sus vínculos y sus actividades, lo cual podrá incidir directa o indirectamente en su equilibrio emocional y desarrollo personal.

\section{Cultura y modo de vida}

Aldous Huxley afirma: "Durante los últimos doscientos o trescientos años, el hombre ha cambiado muy poco anatómica y fisiológicamente. La naturaleza de la capacidad genética que posee un niño actual es esencialmente la misma que poseía un niño habitante de una cueva en el Paleolítico Superior. Pero mientras que el brillante niño de nuestros días puede crecer y convertirse en casi todo -por ejemplo, en un ingeniero presbiteriano, en un pianista marxista o en un profesor de bioquímica agnóstico al que le guste pintar con acuarela- el bebé paleolítico no tenía posibilidad de nada más que de convertirse en cazador o en buscador de alimento, utilizando los instrumentos de piedra más toscos y pensando sobre su limitado mundo de ciénagas y árboles en términos de alguna incierta forma de magia. Los dos bebés, el prehistórico y el actual, son indistinguibles; cada uno de ellos posee todas las potencialidades correspondientes a la variedad de ser humano a la que pertenezcan, 
pero los adultos en torno a los cuales crecerán ambos niños son profundamente distintos porque desarrollarán de manera desigual las potencialidades con las que ha nacido." (A. Huxley, 1965 p.32).

En efecto, es la cultura humana la que puede inventar y promover la diferenciación subjetiva. La cultura que se constituye como la dimensión de transformación, de potencial creador y de creación del hombre, y al mismo tiempo, como el prisma socio-histórico-experiencial mediante el cual, el sujeto conoce y se relaciona con su mundo. Así, la culturalización es precisamente el proceso interpersonal paulatino, diversificado, profundo e imprevisible de asignar, encontrar y/o inventar multívoca y variablemente- sentido, ordenamiento, configuración al conjunto de elementos del mundo. El hombre otorga sentidos al mundo por medio del prisma cultural y la cultura empieza por la acción concreta del hombre en ese mundo ante la posibilidad de transformación, más o menos consciente, del entorno, de sí mismo y de los otros. Es por eso que la configuración de sentidos subjetivos específicos es un proceso concomitante a la realización de acciones por parte del sujeto, siempre en relación a los demás. La cultura se produce cuando se rompe el desenvolvimiento autocrático de lo natural y cuando se deja una impronta, una modificación determinada.

Juan José Guevara Valdés comenta: "La cultura es lo existente, lo formado y lo transformado por el hombre, es también el ir haciendo esa transformación, el proceso de toda la actividad de la especie humana que se objetiva en su creación al tiempo que conquista el mundo natural, lo humaniza; en esta unidad está presente todo el legado material y espiritual de la especie humana. En ella encontramos dos vertientes, la primera la de lo que cada hombre va haciendo y el ir haciendo del conjunto humano, siempre en un tiempo y en un lugar; y la otra vertiente es la que nos conduce a lo que ya está hecho, formado, objetivado, la herencia material y espiritual de toda la existencia humana que nos antecede y ya mencionada, ya que vivimos dentro de una continuidad, después de otros seres, con nuestros contemporáneos y antes que otros, que nos sucederán, aunque ésta continuación no es lineal, ya que lo próximo y lo lejano se acercan y oponen, se cruzan y confunden." (J. Guevara, 1992 p.31). Más adelante este autor señala que a partir de cierto nivel de instrucción y educación, cualquier acción humana tendrá una correspondencia con valores concretos, posibles, viables, previsibles o realizables dentro de la propia existencia personal, y de cierta forma en la continuidad del hacer humano, sin lo cual toda acción se desorganiza y se desorienta y "la realidad se torna tensa, insegura, exigente, imprevisible, interrogante." (Guevara, 1992 p.54).

En concordancia con la reflexión de Guevara Valdés, puede afirmarse que los sentidos que adquiere el individuo respecto a los diferentes aspectos de su vida, provienen por tanto, del prisma de la cultura subjetivizada en la que está inmerso. Se trata de un ejercicio permanente del sujeto, mediante el cual asume diversos contenidos de la cultura, los personaliza y simultáneamente construye sentidos de configuración propia respecto a los mismos, estableciendo así, de hecho, determinadas formas de relación con el entorno y con los demás.

El hombre piensa, habla, siente y se comporta en el ámbito de la intersubjetividad y de la cultura, y en efecto, las vivencias de la relación sujeto-cultura no tienen por qué ser forzosamente extenuantes, destructivas, angustiantes o dolorosas. Tal vez resulta posible reconocer en la persona una disposición, una propensión (una posibilidad) a incorporar las rupturas y las construcciones de la cultura -de forma más o menos armonizada- a su propia existencia desplegada en el devenir de la sociedad que la produce, para lograr precisamente, de manera paulatina, su autoconfiguración irrepetible. Se puede pensar -como señala el propio Guevara Valdés- que se trata de "un progresivo encontrarse a sí mismo, como una continuidad en el pasar del formar al formarse, del hacer al hacerse, al ser uno como persona (...). En este ser uno, hay una vida propia, dada y formada en el 
ser humano, en su relación con los otros, a través de la cultura de su tiempo y lugar, y que lleva en sí el germen del que aparece o salta al éste soy yo y existo como autor de mí mismo, es la primavera de la existencia, que solo brota como continuidad creadora de lo humano y de la intencionalidad de sus antecesores (...)" (Guevara, 1992 p.57).

El individuo que siente depresión o está "estresado", está viviendo tal episodio inmerso en un conjunto de condiciones y relaciones propias, que conjuntamente con las producciones personológicas específicas, hacen de tal desequilibrio un proceso irrepetible que requiere, por parte del psicólogo, un esfuerzo interpretativo detenido y atento, y al unísono, una promoción de los dispositivos al diálogo sostenido y profundo, mediante el cual el sujeto pueda (eventualmente) recomponer o re-construir sus relaciones con el mundo. La salud mental implica -según la idea clásica de Freud- la capacidad personal de amar y trabajar. En esta lógica, Ayestarán y Martínez de Taboada (S. Ayestarán, C. Martínez de Taboada, 1992) plantean que la capacidad de amar puede concebirse como la capacidad de las personas de participar activamente en la construcción de significados sociales en función de mantener una comunicación efectiva tanto a nivel cognitivo como afectivo y motivacional. Por su parte, la capacidad de trabajar puede asociarse con la capacidad de los individuos para comprometerse en la transformación de la realidad social. Se señala que las posiciones de compromiso activo en la transformación del entorno social, afianzan en el sujeto una identidad positiva y promueve actitudes de colaboración en las relaciones interpersonales. La función de la psicología estaría encaminada a desarrollar la capacidad de amar y de trabajar de las personas, por medio de "un aprendizaje grupal centrado en la adquisición de nuevas estrategias para colaborar en la construcción de nuevos significados sociales (mejorar la comunicación) y para participar activamente en el cambio de estructuras sociales (funcionamiento más igualitario dentro de una distrubución adecuada de papeles)." (Ayestarán, Martínez de Taboada, 1992 p.41). La igualdad hace alusión fundamentalmente a la participación en la toma de decisiones del grupo, al hecho de que los miembros del grupo se sientan responsables de su propia evolución y desarrollo, sin que ello signifique la desaparición de los roles específicos ejercidos de manera diferenciada por cada quién.

Un proceso terapéutico en psicología podrá dirigirse entonces -de manera diversa según el caso- a establecer o rehacer procesos de socialización en los individuos con los que se trabaja, y de esta forma, romper con la posible hipóstasis que el mundo (plexo existencial) de esas personas tiene respecto a ciertos vínculos interpersonales y a ciertas configuraciones subjetivo-culturales de carácter disfuncional. En otras palabras, las personas han de ser capaces de afrontar y resolver, recomponer o reabrir por cursos distintos, mediante elaboraciones subjetivo-culturales nuevas, el presunto conflicto entre lo que podríamos llamar el momento interno y el momento externo de su mundo, con vistas a una socialización transformadora y creativa.

La sociedad como el espacio humano de adaptación y de transformación, impone al individuo, en mayor o menor grado y de distinta forma, exigencias y demandas sobre como "debe" vivir. El individuo ha de operar en tales circunstancias. La sociedad entonces -como señala R. Lazarus- es de hecho un elemento configurador de personas y grupos. A través de la cultura social el sujeto otorga diversos matices de sentido a sus interacciones con los demás y con el ambiente. La sociedad y la cultura son el mundo para el recién nacido, constituyen el contexto ineludible en donde habrá de escribirse el texto personal. La cultura de un sistema social y la forma de incorporarse al sujeto, que a su vez expresa necesidades y motivos (en conjunción con las potencialidades activas del propio individuo en la elaboración de tales contenidos) tendrá una importancia capital para la vida afectiva del mismo, máxime al pensar las emociones como producto de interacciones simbólicas. En esta 
lógica, el propio Lazarus escribe: "Dado que la emoción es el resultado de las interpretaciones de la importancia y el significado personal de una transacción, los juicios culturalmente determinados de lo que es importante, deseable, perjudicial o ennoblecedor jugarán un papel muy importante en la determinación de las emociones particulares" (Lazarus, Folkman, 1991 p.249). Por lo mismo, existe una variabilidad cultural que resulta clave en la expresión y el manejo de las emociones y los sentimientos, ya se trate del duelo, la agresividad, el cortejo o el matrimonio. El individuo entonces actúa y siente a través de la fusión de contenidos culturales y experiencia propia en la expresión personalizada de su vida inmersa en vínculos intersubjetivos.

Lazarus, citando a diversos autores tales como Jessor, Asch, Janis entre otros (Lazarus, Folkman, 1991), distingue la dimensión "proximal - distal" la cual se refiere a la "ordenación" que hace el sujeto de sus entornos en relación a la proximidad conceptual, perceptiva, experiencial o interpretativa con los mismos, todo lo cual condiciona su construcción psicológica. Un entorno "proximal" entonces, resulta más significativo para el individuo porque tiene una importancia funcional específica para él. Un entorno "distal" en cambio, es indiferente para la persona y se describe en lenguaje no psicológico o externo. Esta idea puede formularse también en términos del carácter más externo o interno de la cultura personal (R. García, 1999). La cultura personal externa se constituye por contenidos más "distales" para el individuo, mientras que la cultura personal interna se constituye por contenidos más proximales para el sujeto. Frecuentemente, se viven como contenidos "distales" -cultura personal externa- los aspectos sociodemográficos del individuo, como la clase social o las instituciones sociales en general. Los contenidos "proximales" en cambio -cultura personal interna- se viven como ciertas experiencias, percepciones individualizadas, expectativas, pensamientos propios y emociones que, en efecto, constituyen al unísono, la textura de la subjetividad.

En cualquier caso, la relación entre los contenidos socioculturales y las construcciones subjetivas no es una relación de isomorfismo. Las configuraciones subjetivas implican una re-creación activa del mundo externo, una interpretación, una lectura propia, a veces imprevisible que el individuo hace de la cultura que lo rodea, en vínculo con su historicidad, relaciones, temores y deseos, que otorgan sentido único a su comprensión del entorno y a su actuación adaptativa y transformadora en él. Una vez más comenta Lazarus: “(...) cada uno de nosotros tiene una identidad privada, un mundo subjetivo que nunca está del todo abierto a los demás. Cada uno tenemos nuestros propios pensamientos, sentimientos, deseos y metas y nuestra propia conciencia e inconciencia de ello. Lo que cada uno sabe no es exactamente lo mismo que saben los demás y, por tanto, la forma de construir la realidad social varía de un individuo a otro (...)" (Lazarus, Folkman, 1991 p.252).

No basta admitir que determinados contenidos culturales y sociales intervienen en las escisiones de la subjetividad (alienación, alteración), es necesario también, valorar cómo la visión comprensiva e interpretativa del individuo activamente asume, decodifica, codifica y afronta, con actitudes propias, los diversos contenidos socioculturales de relación. Divergencias y/o desfasajes entre la cultura personal interna y la cultura personal externa; necesidades, aspiraciones y deseos que no encuentran o no completan canales de proyección y realización en el mundo que envuelve al sujeto, pueden provocar que ese sujeto se desdibuje. Constituye un proceso dinámico permanente y en cierto momento, un resultado que se observa. Cada ámbito social-cultural, impone a la persona unas $u$ otras demandas o exigencias, favorece expectativas, valores, normas comportamentales, ofrece unos $u$ otros recursos y apoyos sociales que se conjugan de manera irrepetible en el sujeto para que éste se produzca en una dinámica cualitativamente específica de mayor o menor integración. El individuo, 
a lo largo de su desarrollo psicológico, precisamente cultiva de diversa manera, los contenidos sociales en los que crece o se depaupera.

La cultura en la que vive el individuo (expresada también en forma de costumbres, ideales, valores y normas, y en toda elaboración material humana) contrapuntea con él a través de un proceso de introyección y proyección de la que esa cultura es objeto permanente. Así, cada individuo establece a lo largo de su vida una especie de diálogo con el mundo cultural -el modo de vivir- en el cual, por cierto, ese individuo se constituye en sujeto, y dentro del cual, el individuo puede verse muchas veces rebasado en relación a sus capacidades reales de asunción y ejecución. En otras palabras, la construcción de la subjetividad en la cultura, estaría marcada por un proceso de consonanciadisonancia permanente, que también tendría repercusiones ineludibles -aunque diferenciadas- en la calidad de vida de cada persona. El modo de vivir entonces, como conjunto de actividades y relaciones asumidas consciente o inconscientemente por el individuo, así como el sentido psicológico que adquieren, juega un papel muy importante en la posibilidad integral de la salud humana. La potenciación del desarrollo del hombre puede analizarse por tanto, en la articulación de los componentes sistémicos cultura-sociedad-modo de vida-subjetividad-salud psicológica. Además, el individuo puede tener una orientación activa hacia la promoción de su propia cultura personal interna, con la cual se puede abrir espacios de interacción constructiva con los demás y con el mundo y que de hecho, puede constituirse como una cultura de la salud y el desarrollo personal. Desde luego, esta "profilaxis cualitativamente diferente de orientación activo-constructiva y esencialmente humana" como la llama Aldereguía Henríquez- (J. Aldereguía, 1981 p.40) presupone la necesidad de ubicación crítica y transformación de ámbitos enajenantes que muchas veces subyacen en los dispositivos de la convivencia humana.

\section{Referencias}

Aldereguía, J. (1981). El socialismo nacional y la salud. Revista cubana de administración de salud. $7(1), 40$.

Alvaro, J.L., J.R. Torregrosa., A. Garrido. (Comps.). (1992). Influencias sociales y psicológicas de la salud mental. Madrid: Siglo XXI.

Argyle, M. (1992). Efectos del apoyo social derivado de distintas relaciones en la felicidad y la salud mental. En: Alvaro, J.L., J.R. Torregrosa., A. Garrido. (Comps.). Influencias sociales y psicológicas en la salud mental. Madrid: Siglo XXI.

Ayestarán, S., C. Martínez de Taboada. (1992). Posición social y salud mental. En: Alvaro, J.L., J.R. Torregrosa., A. Garrido. (Comps.). Influencias sociales y psicológicas en la salud mental. Madrid: Siglo XXI.

Barrón, A. (1992). Apoyo social y salud mental. En: Alvaro, J.L., J.R. Torregrosa., A. Garrido. (Comps.). Influencias sociales y psicológicas en la salud mental. Madrid: Siglo XXI.

Brown, G.W., T. Harris. (1989). Life events and illness. Nueva York: Guildford Press.

Cochrane, R., R. Sobol (1980). Life stresses and psychological consequences. En: Feldman, P., J. Orford. (Comps.). The social psychology of psychological problems. Chichester: Wiley. 
Friedman, M., R.H. Rosenman. (1974) Type A behavior and your heart. Nueva York: Knof.

García, L. (1988). Estrés psicológico y enfrentamiento. Una actualización del tema. La Habana: Instituto Superior de Ciencias Médicas de La Habana.

García, R. (1999). Cultura y subjetividad. Revista Ethos Educativo. Instituto Michoacano de Ciencias de la Educación. (19) 58-62.

García, R. (1999). Psicología y psicoterapia integrales. Revista Ethos Educativo Instituto Michoacano de Ciencias de la Educación. (21) 88-100.

González, F. (1993). Personalidad Salud y Modo de Vida. México: Universidad Nacional Autónoma de México.

Good, D. (1992). Aproximación a los problemas lingüísticos de los esquizofrénicos desde una perspectiva interactiva. En: Alvaro, J.L., J.R. Torregrosa., A. Garrido. (Comps.). Influencias sociales y psicológicas en la salud mental. Madrid: Siglo XXI.

Guevara, J. (1992). La persona y el tiempo. La Habana: Universidad de La Habana.

Guevara, J., D. Zaldivar. (1989). Psicología clínica e imagen del hombre. La psicología clínica y el desarrollo social. La Habana: Universidad de La Habana, Facultad de Psicología.

Holmes, T. H., R.H. Rahe. (1967). The social readjustmen rating scale. Journal of Psychosomatic Research, (11) $213-218$.

Huxley, A. (1965). Human potentialities. En: Parson, R. E. (1965). Science and Human Affairs. Palo Alto: Science and Behavior Books.

Jenkins C., R. H. Rosenman, J. Zyzanski. (1974). Prediction of clinical coronary heart disease by a test for the coronary - prone behavior pattern. New England Journal of Medicine, (290) 1271-1275.

Lazarus, R.S., S. Folkman. (1991). Estrés y procesos cognitivos. México: Ediciones Martínez Roca.

Mirowsky, J., C.E. Ross. (1989). Social causes of psychological distress. Nueva York: Aldine de Gruyter.

Morales, F. (1999). Psicología de la salud. Conceptos básicos y proyecciones de trabajo. La Habana: Editorial Científico - Técnica.

Orlandini, A. (1994). Sociedad y estrés. Santiago de Cuba: Editorial Oriente.

Ozámiz, A. (1992). Sucesos vitales y trastornos mentales. En: Alvaro, J.L., J.R. Torregrosa., A. Garrido. (Comps.). Influencias sociales y psicológicas en la salud mental. Madrid: Siglo XXI.

Páez, D., J. Adrián., N. Basabe. (1992). Balanza de afectos, dimensiones de la afectividad y emociones: una aproximación sociológica a la salud mental. En: Alvaro, J.L., J.R. Torregrosa., A. Garrido. (Comps.). Influencias sociales y psicológicas en la salud mental. Madrid: Siglo XXI.

Roca, M., M. Pérez. (1999). Apoyo social: su significación para la salud humana. La Habana: Editorial Félix Varela. 
Rochester, Martin. (1977). Crazy Talk. Nueva York: Wiley.

Sánchez, A. (1991).Psicología comunitaria. Bases conceptuales y operativas. Métodos de intervención. Barcelona: P.P.U.

Schütz, A. (1973). La estructuras del mundo de la vida. Buenos Aires: Amorrourtu

Weiss, R.S. (1974). The Provisions of Social Relationships. En: Rubin, Z. (1974). Doing into others. Nueva Jersey: Prentice Hall.

Zaldívar, D. (1996). Conocimiento y dominio del estrés. La Habana: Editorial Científico - Técnica.

\section{Formato de citación}

García, Raúl. (2005). Notas sobre la noción de salud y la reflexión cultural en la psicología. Athenea Digital, 9. Disponible en http://antalya.uab.es/athenea/num9/Garcia.pdf.

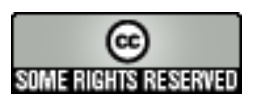

Este texto está protegido por una licencia Creative Commons.

Usted es libre de copiar, distribuir y comunicar públicamente la obra bajo las siguientes condiciones:

Reconocimiento: Debe reconocer y citar al autor original.

No comercial. No puede utilizar esta obra para fines comerciales.

Sin obras derivadas. No se puede alterar, transformar, o generar una obra derivada a partir de esta obra.

\section{$\underline{\text { Resumen de licencia }}$}

$\underline{T e x t o ~ c o m p l e t o ~ d e ~ l a ~ l i c e n c i a ~}$ 